\title{
ESSENTIAL COMMUTANTS OF SEMICROSSED PRODUCTS
}

\author{
KEI HASEGAWA
}

\begin{abstract}
Let $\alpha: G \curvearrowright M$ be a spatial action of countable abelian group on a "spatial" von Neumann algebra $M$ and $S$ be its unital subsemigroup with $G=S^{-1} S$. We explicitly compute the essential commutant and the essential fixed-points, modulo the Schatten $p$-class or the compact operators, of the w*-semicrossed product of $M$ by $S$ when $M^{\prime}$ contains no non-zero compact operators. We also prove a weaker result when $M$ is a von Neumann algebra on a finite dimensional Hilbert space and $(G, S)=\left(\mathbb{Z}, \mathbb{Z}_{+}\right)$, which extends a famous result due to Davidson (1977) for the classical analytic Toeplitz operators.
\end{abstract}

\section{INTRODUCTION}

Let $A$ be a (not necessarily self-adjoint) operator algebra on a Hilbert space $\mathcal{H}$. We denote by $\mathfrak{S}_{p}=\mathfrak{S}_{p}(\mathcal{H})$ the Schatten $p$-class operators on $\mathcal{H}$ with $1 \leq p<\infty$ and by $\mathfrak{S}_{\infty}=\mathfrak{S}_{\infty}(\mathcal{H})$ the compact operators $\mathcal{K}=\mathcal{K}(\mathcal{H})$ on $\mathcal{H}$. We also denote by $\mathcal{I}(A)$ the set of all isometries in $A$. In this paper we investigate the following two sets:

$$
\begin{aligned}
& \operatorname{Esscom}_{p}(A)=\left\{X \in \mathcal{B}(\mathcal{H}) \mid a X-X a \in \mathfrak{S}_{p}(\mathcal{H}) \text { for } a \in A\right\}, \\
& \operatorname{Essfix}_{p}(A)=\left\{X \in \mathcal{B}(\mathcal{H}) \mid v^{*} X v-X \in \mathfrak{S}_{p}(\mathcal{H}) \text { for } v \in \mathcal{I}(A)\right\},
\end{aligned}
$$

called the essential commutant and the essential fixed-points of $A$ modulo the *ideal $\mathfrak{S}_{p}$, respectively. Clearly, $\operatorname{Esscom}_{p}(A)$ is contained in $\operatorname{Essfix}_{p}(A)$, and these two sets coincide when $A$ is a $\mathrm{C}^{*}$-algebra which contains the identity operator, since any unital $\mathrm{C}^{*}$-algebra is the linear span of its unitary elements. Johnson and Parrott [8] and Popa 13 proved that $\operatorname{Esscom}_{\infty}(A)=A^{\prime}+\mathcal{K}$ holds when $A$ is a von Neumann algebra, and Hoover [7] proved that $\operatorname{Esscom}_{p}(A)=A^{\prime}+\mathfrak{S}_{p}$ when $A$ is a $\mathrm{C}^{*}$-algebra but $p \neq \infty$. On the other hand, for a non-self-adjoint algebra these two sets do not coincide in general, and thus the computation of them is non-trivial. Such non-trivial works, among others, are $\operatorname{Esscom}_{\infty}\left(T\left(H^{\infty}\right)\right)=T\left(H^{\infty}+C\right)+\mathcal{K}\left(H^{2}\right)$ due to Davidson 2 and $\operatorname{Essfix}_{\infty}\left(T\left(H^{\infty}\right)\right)=T\left(L^{\infty}\right)+\mathcal{K}\left(H^{2}\right)$ due to Xia [16, where $T\left(H^{\infty}\right), T\left(H^{\infty}+C\right)$, and $T\left(L^{\infty}\right)$ are the sets of all Toeplitz operators on the Hardy space $H^{2}=H^{2}(\mathbb{T})$ whose symbols are in the bounded Hardy space $H^{\infty}=H^{\infty}(\mathbb{T})$, the Douglas algebra $H^{\infty}+C$ (with $C=C(\mathbb{T})$ ), and $L^{\infty}=L^{\infty}(\mathbb{T})$, respectively. See [3, Chapter 6,7] for those terminologies.

In this paper, we study the essential commutant and the essential fixed-points for $\mathrm{w}^{*}$-semicrossed products. The notation below follows $[9$. Let $M$ be a von Neumann algebra on a Hilbert space $\mathcal{H}, G$ be a countable abelian group acting on $M$ by $\alpha$, and $S$ be a subsemigroup of $G$ which contains the unit of $G$ and generates $G$. We also assume that the action $\alpha: G \curvearrowright M$ is spatial, that is, $\alpha$ is implemented by unitary operators $\left\{u_{g} \mid g \in G\right\}$ on $\mathcal{H}$. We denote by the same symbol $\alpha$ the action of $G$ on $M^{\prime}$ implemented by $\left\{u_{g} \mid g \in G\right\}$. Then we 
can construct the left and the right $\mathrm{w}^{*}$-crossed products $G \bar{\ltimes}_{\alpha} M$ and $M^{\prime} \bar{\rtimes}_{\alpha} G$ on $\mathbb{L}^{2}:=\mathcal{H} \otimes \ell^{2}(G)$ with $\left(G \bar{\ltimes}_{\alpha} M\right)^{\prime}=M^{\prime} \bar{\rtimes}_{\alpha} G$. The left and the right reduced w*semicrossed products $S \bar{\ltimes}_{\alpha} M$ and $M \bar{\rtimes}_{\alpha} S$ are constructed as $\sigma$-weakly closed subalgebras of $G \bar{\ltimes}_{\alpha} M$ and $M \bar{\rtimes}_{\alpha} G$, respectively. Let $P$ denote the orthogonal projection from $\mathbb{L}^{2}$ onto $\mathbb{H}^{2}:=\mathcal{H} \otimes \ell^{2}(S)$ and define the Toeplitz map $T: \mathcal{B}\left(\mathbb{L}^{2}\right) \rightarrow \mathcal{B}\left(\mathbb{H}^{2}\right)$ by $T_{X}:=\left.P X\right|_{\mathbb{H}^{2}}$ for $X \in \mathcal{B}\left(\mathbb{L}^{2}\right)$. The left and the right $\mathrm{w}^{*}$-semicrossed products $S \overline{\times}_{\alpha} M$ and $M \overline{\times}_{\alpha} S$ are constructed as $\sigma$-weakly closed subalgebras of $\mathcal{B}\left(\mathbb{H}^{2}\right)$, and in fact, coincide with $T\left(S \bar{\ltimes}_{\alpha} M\right)$ and $T\left(M \bar{\rtimes}_{\alpha} S\right)$, respectively (see Proposition 2.6), where for a subset $\mathfrak{F}$ of $\mathcal{B}\left(\mathbb{L}^{2}\right)$ the $T(\mathfrak{F})$ stands for $\left\{T_{F} \mid F \in \mathfrak{F}\right\}$. In the typical case of $(M, \mathcal{H}, G, S)=\left(\mathbb{C}, \mathbb{C}, \mathbb{Z}, \mathbb{Z}_{+}\right)$, the "left" and the "right" algebras $\left(G \bar{\ltimes}_{\alpha} M, S \bar{\ltimes}_{\alpha} M, S \overline{\times}_{\alpha} M\right)$ and $\left(M \bar{\rtimes}_{\alpha} G, M \bar{\rtimes}_{\alpha} S, M \overline{\times}_{\alpha} S\right)$ coincide and become $\left(L^{\infty}, H^{\infty}, T\left(H^{\infty}\right)\right)$. The concept of $\mathrm{w}^{*}$-semicrossed products and reduced ones were introduced by Kakariadis [9] for $\sigma$-weakly closed operator algebras and their normal endomorphisms and for von Neumann algebras and their automorphisms, i.e., the case of $(G, S)=\left(\mathbb{Z}, \mathbb{Z}_{+}\right)$, respectively. The latter coincides with the adjoint of analytic crossed products studied by McAsey, Muhly, and Saito [11. Toeplitz operators associated with analytic crossed products were studied by Saito [14 and the algebras of analytic Toeplitz operators in this sense essentially coincide with $\mathrm{w}^{*}$-semicrossed products as above.

In Section 2 we define these objects and prove some elementary properties. When $M^{\prime}$ contains no non-zero compact operators, we can explicitly compute $\operatorname{Esscom}_{p}\left(S \overline{\times}_{\alpha} M\right)$ and $\operatorname{Essfix}_{p}\left(S \overline{\times}_{\alpha} M\right)$ as follows.

Theorem 1.1. Let $M \subset \mathcal{B}(\mathcal{H})$ be a von Neumann algebra, $\alpha: G \curvearrowright M$ a spatial action of a discrete countable abelian group, and $S$ be a subsemigroup of $G$ which contains the unit of $G$ and generates $G$. If $M^{\prime}$ contains no non-zero compact operators, then

$\operatorname{Essfix}_{p}\left(S \overline{\times}_{\alpha} M\right)=T\left(M^{\prime} \bar{\rtimes}_{\alpha} G\right)+\mathfrak{S}_{p}\left(\mathbb{H}^{2}\right), \operatorname{Esscom}_{p}\left(S \overline{\times}_{\alpha} M\right)=M^{\prime} \overline{\times}_{\alpha} S+\mathfrak{S}_{p}\left(\mathbb{H}^{2}\right)$ hold for every $1 \leq p \leq \infty$.

Corollary 1.2. The same assertion of Theorem 1.1 holds when $\mathcal{H}$ is the standard Hilbert space $L^{2}(M)$ and $M$ is either diffuse, $\mathcal{B}\left(\ell^{2}\right)$ of infinite dimension, or a (possibly infinite) direct sum of them.

An another immediate corollary of Theorem 1.1 is the double commutant theorem for $S \overline{\times}_{\alpha} M$ and $S \overline{\times}_{\alpha} M^{\prime}$ in the Calkin algebra under the assumption that $M \cap \mathcal{K}=M^{\prime} \cap \mathcal{K}=\{0\}$. The proofs of Theorem 1.1 and Corollary 1.2 will be given in Section 3. In Section 4, we consider the case that $\mathcal{H}$ is finite dimensional (and hence so is $M)$ and $(G, S)=\left(\mathbb{Z}, \mathbb{Z}_{+}\right)$, and can prove the following theorem:

Theorem 1.3. Let $M$ be a von Neumann algebra on a finite dimensional Hilbert space $\mathcal{H}$ and $\alpha$ be $a$ *-automorphism on $M$ implemented by a unitary operator on $\mathcal{H}$. Then we have

$$
\operatorname{Esscom}_{p}\left(\mathbb{Z}_{+} \bar{x}_{\alpha} M\right) \subset T\left(M^{\prime} \bar{\rtimes}_{\alpha} \mathbb{Z}\right)+\mathfrak{S}_{p}\left(\mathbb{H}^{2}\right) \quad \text { for } 1 \leq p \leq \infty .
$$

This theorem is nothing but [2, Theorem 2] for the classical analytic Toeplitz operators by specializing $M=\mathbb{C}$ and $p=\infty$, though our proof is a bit improved with the help of several ideas in [12, [16]. The reason why we do not consider the essential fixed-points is that there is a technical obstruction to translating the argument in [16] into our setting (see Remark[4.7). In the final section, we examine 
whether or not the assertion of Theorem 1.3 still holds when $M$ is an arbitrary von Neumann algebra of standard form.

\section{Preliminaries}

We begin with a small remark.

Remark 2.1. For a given $v \in \mathcal{I}(A)$ we consider the unital completely positive map $\Psi_{v}: X \mapsto v^{*} X v$ on $\mathcal{B}(\mathcal{H})$. Then $\operatorname{Essfix}_{p}(A)$ is nothing but the set of operators "fixed modulo $\mathfrak{S}_{p}$ " by $\left\{\Psi_{v} \mid v \in \mathcal{I}(A)\right\}$. It is immediate to see that $\operatorname{Esscom}_{p}(A) \subset$ $\operatorname{Essfix}_{p}(A)$. Moreover, these two sets coincide if $A$ is a $\mathrm{C}^{*}$-algebra, since any $\mathrm{C}^{*}$ algebra is generated by its unitary elements. By [8, 13] and [7] any von Neumann algebra $M \subset \mathcal{B}(\mathcal{H})$ enjoys $\operatorname{Esscom}_{p}(M)=\operatorname{Essfix}_{p}(M)=M^{\prime}+\mathfrak{S}_{p}$ for every $1 \leq p \leq$ $\infty$.

Next, let us recall $\mathrm{w}^{*}$-semicrossed products and Toeplitz maps associated with them. Our notation and formulation follow 9. Remark that those do not completely agree with the usual ones for crossed products. Let $M \subset \mathcal{B}(\mathcal{H})$ be a von Neumann algebra and $G$ be a countable discrete abelian group. Assume that we have a spatial action $\alpha: G \curvearrowright M$ implemented by a unitary representation $u: G \ni g \mapsto u_{g} \in \mathcal{B}(\mathcal{H})$, i.e., we have $\alpha_{g}(x)=u_{g} x u_{g}^{*}$ for every $x \in M$ and $g \in G$. Since $G$ acts on $M^{\prime}$ too by $\operatorname{Ad} u_{g}$, we also denote by the same symbol $\alpha_{g}$ the automorphism $\operatorname{Ad} u_{g}$ on $M^{\prime}$. Let $G \ni g \mapsto \lambda_{g} \in \mathcal{B}\left(\ell^{2}(G)\right)$ be the left regular representation of $G$ and $e_{g} \in \mathcal{B}\left(\ell^{2}(G)\right)$ be the orthogonal projection onto $\mathbb{C} \delta_{g}$. Set $\mathbb{L}^{2}:=\mathcal{H} \otimes \ell^{2}(G)$ and define representations $\pi: M \rightarrow \mathcal{B}\left(\mathbb{L}^{2}\right)$ and $\lambda(\cdot), \rho(\cdot): G \rightarrow \mathcal{B}\left(\mathbb{L}^{2}\right)$ by

$$
\pi(x)=\sum_{g \in G} \alpha_{g}(x) \otimes e_{g}, \quad \lambda(g)=1 \otimes \lambda_{g}, \quad \rho(g)=u_{g}^{*} \otimes \lambda_{g}
$$

for $x \in M$ and $g \in G$. The left and the right $w^{*}$-crossed products of $M$ by $G$ are defined to be the von Neumann algebras $G \bar{\ltimes}_{\alpha} M=\pi(M) \vee\{\lambda(g) \mid g \in G\}^{\prime \prime}$ and $M \bar{\rtimes}_{\alpha} G:=M \otimes \mathbb{C} 1 \vee\{\rho(g) \mid g \in G\}^{\prime \prime}$, respectively. It is well-known that $\left(G \bar{\ltimes}_{\alpha} M\right)^{\prime}=M^{\prime} \bar{\rtimes}_{\alpha} G$ (see e.g. [15, Theorem 1.21]). We also note that when $M$ is of standard form, each action $\alpha: G \curvearrowright M$ is spatial thanks to [4, Theorem 3.2].

Definition 2.1 (Kakariadis 9]). Let $M \subset \mathcal{B}(\mathcal{H})$ and $\alpha: G \curvearrowright M$ be as above. For a given subsemigroup $S$ of $G$ which contains the unit $\iota$ of $G$ and generates $G$, the left and the right reduced $w^{*}$-semicrossed product $S \bar{\ltimes}_{\alpha} M$ and $M \bar{\rtimes}_{\alpha} S$ of $M$ by $S$ are defined to be the $\sigma$-weakly closed subalgebras of $G \bar{\ltimes}_{\alpha} M$ and $M \bar{\rtimes}_{\alpha} G$ generated by $\pi(M)$ and $\{\lambda(s) \mid s \in S\}$ and by $M \otimes \mathbb{C} 1$ and $\{\rho(s) \mid s \in S\}$, respectively.

Let $P$ be the projection onto $\mathbb{H}^{2}:=\mathcal{H} \otimes \ell^{2}(S) \subset \mathbb{L}^{2}$. The Toeplitz map $T$ : $\mathcal{B}\left(\mathbb{L}^{2}\right) \rightarrow \mathcal{B}\left(\mathbb{H}^{2}\right)$ is defined by $T_{X}:=\left.P X\right|_{\mathbb{H}^{2}}$ for $X \in \mathcal{B}\left(\mathbb{L}^{2}\right)$. The left and the right $w^{*}$-semicrossed product $S \overline{\times}_{\alpha} M$ and $M \overline{\times}_{\alpha} S$ of $M$ by $S$ are defined to be $\sigma$-weakly closed subalgebras of $\mathcal{B}\left(\mathbb{H}^{2}\right)$ generated by $T(\pi(M))$ and $\left\{T_{\lambda(s)} \mid s \in S\right\}$ and by $M \otimes \mathbb{C} 1_{\ell^{2}(S)}$ and $\left\{T_{\rho(s)} \mid s \in S\right\}$, respectively. Here for a subset $\mathfrak{F}$ of $\mathcal{B}\left(\mathbb{L}^{2}\right)$ the $T(\mathfrak{F})$ stands for $\left\{T_{F} \mid F \in \mathfrak{F}\right\}$.

Remark 2.2. Since $T$ is normal and multiplicative on $S \bar{\ltimes}_{\alpha} M$ and $M \bar{\rtimes}_{\alpha} S$, one has $T\left(S \bar{\ltimes}_{\alpha} M\right) \subset S \overline{\times}_{\alpha} M \subset{\overline{T\left(S \bar{\ltimes}_{\alpha} M\right)}}^{\sigma-\mathrm{w}}$ and $T\left(M \bar{\rtimes}_{\alpha} S\right) \subset M \overline{\times}_{\alpha} S \subset{\overline{T\left(M \bar{\rtimes}_{\alpha} S\right.}}^{\sigma \text {-w }}$. Moreover, for any $x, z \in\left(S \bar{\ltimes}_{\alpha} M\right) \cup\left(M \bar{\rtimes}_{\alpha} S\right)$ and $Y \in \mathcal{B}\left(\mathbb{L}^{2}\right)$, we have $T_{x}^{*} T_{Y} T_{z}=$ $T_{x^{*} Y z}$. We also note that $P$ is in $\pi(M)^{\prime}$, and hence one has

$$
\operatorname{Essfix}_{p}(T(\pi(M)))=\operatorname{Esscom}_{p}(T(\pi(M)))=T\left(\pi(M)^{\prime}\right)+\mathfrak{S}_{p} .
$$


We will use these facts throughout.

Remark 2.3. Define a unitary operator $W:=\sum_{g \in G} u_{g} \otimes e_{g}$ on $\mathbb{L}^{2}$. It is easily seen that $W^{*} \pi(x) W=x \otimes 1$ and $W^{*} \lambda(g) W=\rho(g)$ for $x \in M$ and $g \in G$. Moreover, since $W$ commutes with $P, \hat{W}:=T_{W}$ is also unitary on $\mathbb{H}^{2}$, and hence one has $\hat{W}^{*} T_{\pi(x)} \hat{W}=x \otimes 1_{\ell^{2}(S)}$ and $\hat{W}^{*} T_{\lambda(g)} \hat{W}=T_{\rho(g)}$ for $x \in X$ and $g \in G$. Therefore, the "left" and the "right" algebras are unitarily equivalent.

Proposition 2.4. Let $M, G, S, \alpha$ be as in Definition 2.1. Then we have $S \bar{\ltimes}_{\alpha} M=$ $\left\{x \in G \bar{\ltimes}_{\alpha} M \mid x\left(1 \otimes e_{\iota}\right)=P x\left(1 \otimes e_{\iota}\right)\right\}$ and $M \bar{\rtimes}_{\alpha} S=\left\{x \in M \bar{\rtimes}_{\alpha} G \mid x\left(1 \otimes e_{\iota}\right)=\right.$ $\left.\operatorname{Px}\left(1 \otimes e_{\iota}\right)\right\}$.

Proof. Clearly, $S \bar{\ltimes}_{\alpha} M$ is contained in $\left\{x \in G \bar{\ltimes}_{\alpha} M \mid x\left(1 \otimes e_{\iota}\right)=P x\left(1 \otimes e_{\iota}\right)\right\}$. Let $\hat{G}$ be the dual group of $G$, and $\hat{\alpha}: \hat{G} \rightarrow \operatorname{Aut}\left(G \bar{\ltimes}_{\alpha} M\right)$ the dual action. By [10, Corollary 4.3.2], $S \bar{\ltimes}_{\alpha} M$ is the spectral subspace of $G \bar{\ltimes}_{\alpha} M$ associated with $S$ by the dual action, and we denote it by $\left(G \bar{\ltimes}_{\alpha} M\right)^{\hat{\alpha}}(S)$. It is easily seen that $\left(G \bar{\ltimes}_{\alpha} M\right)^{\hat{\alpha}}(S)$ contains $\left\{x \in G \bar{\ltimes}_{\alpha} M \mid x\left(1 \otimes e_{\iota}\right)=P x\left(1 \otimes e_{\iota}\right)\right\}$. By the preceding remark, one has $M \bar{\rtimes}_{\alpha} S=\left\{x \in M \bar{\rtimes}_{\alpha} G \mid x\left(1 \otimes e_{\iota}\right)=P x\left(1 \otimes e_{\iota}\right)\right\}$.

Recall that a semigroup $S$ is right amenable if $S$ has a right invariant mean, that is, there exists a state $\psi$ on $\ell^{\infty}(S)$ which satisfies that $\psi(f)=\psi\left(r_{s} f\right)$ for $f \in \ell^{\infty}(S)$ and $s \in S$, where $r_{s} f(t)=f(t s), t \in S$. It is known, see [5, Theorem 17.5], that every abelian semigroup is (right) amenable.

Proposition 2.5. If $S$ is a right amenable semigroup and $\sigma: S \rightarrow \mathcal{B}(\mathcal{H})$ is a unitary representation, then $\{\sigma(s) \mid s \in S\}^{\prime} \cap \overline{\mathrm{Co}}^{\mathrm{w}}\left\{\sigma(s)^{*} x \sigma(s) \mid s \in S\right\} \neq \emptyset$ for every $x \in \mathcal{B}(\mathcal{H})$.

Proof. Let $\psi$ be a right invariant mean on $S$. Fix $x \in \mathcal{B}(\mathcal{H})$. For $\xi \in \mathfrak{S}_{1}(\mathcal{H})$, define $f_{\xi} \in \ell^{\infty}(S)$ by $f_{\xi}(s)=\operatorname{Tr}\left(\sigma(s)^{*} x \sigma(s) \xi\right), s \in S$. Then there exists $y \in$ $\mathcal{B}(\mathcal{H}) \cong \mathfrak{S}_{1}(\mathcal{H})^{*}$ such that $\operatorname{Tr}(y \xi)=\psi\left(f_{\xi}\right)$ for $\xi \in \mathfrak{S}_{1}(\mathcal{H})$. Since $f_{\sigma(s) \xi \sigma(s)^{*}}=r_{s} f_{\xi}$ for $s \in S$ and $\psi$ is right invariant, we have $\operatorname{Tr}\left(\sigma(s)^{*} y \sigma(s) \xi\right)=\psi\left(f_{\sigma(s) \xi \sigma(s)^{*}}\right)=$ $\psi\left(f_{\xi}\right)=\operatorname{Tr}(y \xi)$, which implies that $y \in\{\sigma(s) \mid s \in S\}^{\prime}$. Suppose that $y \notin$ $\overline{\mathrm{CO}}^{\mathrm{w}}\left\{\sigma(s)^{*} x \sigma(s) \mid s \in S\right\}$. By the Hahn-Banach separation theorem, there exist $\xi \in \mathfrak{S}_{1}(\mathcal{H})$ and a constant $c \in \mathbb{R}$ such that $\operatorname{Re} \psi\left(f_{\xi}\right)=\operatorname{Re} \operatorname{Tr}(y \xi)<c \leq$ $\operatorname{Re} \operatorname{Tr}\left(\sigma(s)^{*} x \sigma(s)\right)=\operatorname{Re} f_{\xi}(s)$ for $s \in S$. However, by the Kreǐn-Mil'man theorem, $\psi$ falls in the weak* closed convex hull of $S \subset \ell^{\infty}(S)^{*}$, a contradiction.

The following proposition gives us a Brown-Halmos type criterion ([1, Theorem 6 and 7]).

Proposition 2.6. Let $M \subset \mathcal{B}(\mathcal{H}), S \subset G, \alpha$ be as in Definition 2.1. Then the following are true.

(i) For a given $X \in \mathcal{B}\left(\mathbb{H}^{2}\right), X$ falls in $T\left(M^{\prime} \bar{\rtimes}_{\alpha} G\right)$ (resp. $T\left(G \bar{\ltimes}_{\alpha} M^{\prime}\right)$ ) if and only if $X$ commutes with $T(\pi(M))$ (resp. $M \otimes \mathbb{C} 1_{\ell^{2}(S)}$ ) and satisfies that $T_{\lambda(s)}^{*} X T_{\lambda(s)}=X\left(\right.$ resp. $\left.T_{\rho(s)}^{*} X T_{\rho(s)}=X\right)$ for every $s \in S$.

(ii) $T\left(M^{\prime} \bar{\rtimes}_{\alpha} S\right)=M^{\prime} \overline{\times}_{\alpha} S=\left(S \overline{\times}_{\alpha} M\right)^{\prime}$ and $T\left(S \bar{\ltimes}_{\alpha} M^{\prime}\right)=S \overline{\times}_{\alpha} M^{\prime}=\left(M \overline{\times}_{\alpha} S\right)^{\prime}$.

Proof. Let $X \in T(\pi(M))^{\prime}$ be arbitrarily chosen in such a way that $T_{\lambda(s)}^{*} X T_{\lambda(s)}=X$ for every $s \in S$. Since $T(\pi(M))^{\prime}=T\left(\pi(M)^{\prime}\right)$, there exists $x \in \pi(M)^{\prime}$ such that $X=T_{x}$. By Proposition 2.5, we find $y$ in $\overline{\operatorname{co}}^{\mathrm{w}}\left\{\lambda(s)^{*} \operatorname{Px} P \lambda(s) \mid s \in S\right\} \cap\{\lambda(s) \mid s \in$ $S\}^{\prime}$. Note that $y$ is in $M^{\prime} \bar{\rtimes}_{\alpha} G=\left(G \bar{\ltimes}_{\alpha} M\right)^{\prime}=\pi(M)^{\prime} \cap\{\lambda(g) \mid g \in G\}^{\prime}$ since PxP is 
in $\pi(M)^{\prime}$, the $\lambda(s), s \in S$, normalize $\pi(M)^{\prime}$, and $S$ generates $G$. Since the Toeplitz map $T$ is $\sigma$-weakly continuous, one has $T_{y} \in \overline{\mathrm{co}}^{\mathrm{w}}\left\{T_{\lambda(s)}^{*} X T_{\lambda(s)} \mid s \in S\right\}=\{X\}$, which implies that $X=T_{y} \in T\left(M^{\prime} \bar{\rtimes}_{\alpha} G\right)$. Conversely, let $x$ be in $M^{\prime} \bar{\rtimes}_{\alpha} G \subset \pi(M)^{\prime}$. Then $T_{x} \in T\left(\pi(M)^{\prime}\right)=T(\pi(M))^{\prime}$. For any $s \in S$, we have $T_{\lambda(s)}^{*} T_{x} T_{\lambda(s)}=T_{\lambda(s)^{*} x \lambda(s)}=$ $T_{x}$, which implies (i).

To see (ii) it suffices to prove that $\left(S \overline{\times}_{\alpha} M\right)^{\prime}=T\left(M^{\prime} \bar{\rtimes}_{\alpha} S\right)$ by Remark 2.2. It is immediate to see that $T\left(M^{\prime} \bar{\rtimes}_{\alpha} S\right) \subset\left(S \overline{\times}_{\alpha} M\right)^{\prime}$. Conversely, let $Y \in\left(S \overline{\times}_{\alpha} M\right)^{\prime}$ be arbitrary. By the preceding paragraph, there exists $a \in M^{\prime} \bar{\rtimes}_{\alpha} G$ such that $Y=T_{a}$. By the assumption that $G=S^{-1} S$, for each $g \in G \backslash S$ there exist $s, t \in S$ such that $g=s^{-1} t$. Since $a$ commutes with $\lambda(s), s \in S$, we have

$$
\begin{aligned}
\left\langle a \xi \otimes \delta_{\iota}, \eta \otimes \delta_{g}\right\rangle & =\left\langle(1-P) a P \xi \otimes \delta_{\iota}, \eta \otimes \delta_{s^{-1} t}\right\rangle=\left\langle P \lambda(s)(1-P) a P \xi \otimes \delta_{\iota}, \eta \otimes \delta_{t}\right\rangle \\
& =\left\langle\left[T_{a}, T_{\lambda(s)}\right] \xi \otimes \delta_{\iota}, \eta \otimes \delta_{t}\right\rangle=0
\end{aligned}
$$

for every $\xi, \eta \in \mathcal{H}$, and hence $\left(1 \otimes e_{g}\right) a\left(1 \otimes e_{\iota}\right)=0$. Therefore, $a$ falls in $M^{\prime} \bar{\rtimes}_{\alpha} S$ by Proposition 2.4 .

\section{Proof of Theorem 1.1}

Throughout this section, let $M \subset \mathcal{B}(\mathcal{H})$ denote a von Neumann algebra, $\alpha: G \curvearrowright$ $N$ a spatial action of discrete countable abelian group, and $S$ a subsemigroup of $G$ which contains the unit $\iota$ of $G$ and generates $G$.

Lemma 3.1. If $M^{\prime}$ (resp. $\left.M\right)$ contains no non-zero compact operators, then so does $\pi(M)^{\prime}\left(\right.$ resp. $\left.\left(M^{\prime} \otimes \mathbb{C} 1\right)^{\prime}\right)$.

Proof. Assume that $M^{\prime} \cap \mathcal{K}=\{0\}$. By Remark 2.3 it suffices to prove that $(M \otimes$ $\mathbb{C} 1)^{\prime} \cap \mathcal{K}\left(\mathbb{L}^{2}\right)=\{0\}$. Let $K \in(M \otimes \mathbb{C} 1)^{\prime} \cap \mathcal{K}\left(\mathbb{L}^{2}\right)$ be arbitrary chosen. Since $(M \otimes \mathbb{C} 1)^{\prime}=M^{\prime} \bar{\otimes} \mathcal{B}\left(\ell^{2}(G)\right)$, the $\left.\left(1 \otimes \lambda_{g}^{*} e_{g}\right) K\left(1 \otimes e_{h} \lambda_{h}\right)\right|_{\mathcal{H} \otimes \mathbb{C} \delta_{\iota}}$ falls in $\left(M^{\prime} \otimes \mathbb{C} e_{\iota}\right) \cap$ $\mathcal{K}\left(\mathcal{H} \otimes \mathbb{C} \delta_{\iota}\right) \cong M^{\prime} \cap \mathcal{K}(\mathcal{H})=\{0\}$ for every $g, h \in G$, which implies $K=0$.

Lemma 3.2. The restrictions of the Toeplitz map to $\{\lambda(g) \mid g \in G\}^{\prime}$ and $\{\rho(h) \mid h \in$ $G\}^{\prime}$ are isometries. Consequently, every isometry in $S \overline{\times}_{\alpha} M$ and $M \overline{\times}_{\alpha} S$ is of the form $T_{v}$ with some isometry $v$ in $S \bar{\ltimes}_{\alpha} M$ and $M \bar{\rtimes}_{\alpha} S$, respectively.

Proof. Firstly, we prove that the restriction of $T$ to $\{\lambda(g) \mid g \in G\}^{\prime}$ is injective. Let $x \in\{\lambda(g) \mid g \in G\}^{\prime}$ be chosen in such a way that $T_{x}=0$. Since $S$ generates $G$ and $G$ is abelian, for each $g, h \in G$ there exist $s, s^{\prime}, t, t^{\prime} \in S$ such that $g=t^{-1} s$ and $h=t^{\prime-1} s^{\prime}$. Since $x$ commutes with $\lambda(g), g \in G$, one has

$$
\begin{aligned}
\left\langle x\left(\xi \otimes \delta_{t^{-1} s}\right), \eta \otimes \delta_{t^{\prime-1} s^{\prime}}\right\rangle & =\left\langle x\left(\xi \otimes \lambda_{t}^{*} \delta_{s}\right), \eta \otimes \lambda_{t^{\prime}}^{*} \delta_{s^{\prime}}\right\rangle=\left\langle x\left(\xi \otimes \lambda_{t^{\prime}} \delta_{s}\right), \eta \otimes \lambda_{t} \delta_{s^{\prime}}\right\rangle \\
& =\left\langle T_{x}\left(\xi \otimes \delta_{t^{\prime} s}\right), \eta \otimes \delta_{t s^{\prime}}\right\rangle=0
\end{aligned}
$$

for every $\xi, \eta \in \mathcal{H}$, and hence $x=0$. By Proposition 2.5 there exists $y \in$ $\overline{\mathrm{CO}}^{\mathrm{w}}\left\{\lambda(s)^{*} P x P \lambda(s) \mid s \in S\right\} \cap\{\lambda(g) \mid g \in G\}^{\prime}$. Note that $\|y\| \leq\left\|T_{x}\right\|$. Since $P \lambda(s) P=\lambda(s) P$ for $s \in S$, one has

$$
T_{y} \in \overline{\mathrm{CO}}^{\mathrm{w}}\left\{T_{\lambda(s)}^{*} T_{x} T_{\lambda(s)} \mid s \in S\right\}=\overline{\mathrm{CO}}^{\mathrm{w}}\left\{T_{\lambda(s)^{*} x \lambda(s)} \mid s \in S\right\}=\left\{T_{x}\right\} .
$$

Since $T$ is injective on $\{\lambda(g) \mid g \in G\}^{\prime}$, it follows that $x=y$. Thus $\|x\| \leq\left\|T_{x}\right\| \leq$ $\|x\|$, and hence the restriction of $T$ to $\{\lambda(g) \mid g \in G\}^{\prime}$ is isometric.

To see the second assertion let $V \in \mathcal{I}\left(S \overline{\times}_{\alpha} M\right)$ be arbitrary. By Proposition 2.6 we find $v \in S \bar{\ltimes}_{\alpha} M$ in such a way that $V=T_{v}$. Then one has $1=V^{*} V=T_{v}^{*} T_{v}=$ $T_{v^{*} v}$. Since $T$ is injective on $S \bar{\ltimes}_{\alpha} M \subset\{\rho(g) \mid g \in G\}^{\prime}, v$ itself must be isometry. Similarly, one can prove the same assertion for $\{\rho(g) \mid g \in G\}^{\prime}$ and $M \overline{\times}_{\alpha} S$. 
K. HASEGAWA

By the preceding lemma, it is immediate to see that $T\left(M^{\prime} \bar{\rtimes}_{\alpha} G\right)+\mathfrak{S}_{p}$ is contained in $\operatorname{Essfix}_{p}\left(S \overline{\times}_{\alpha} M\right)$. Hence the next theorem completes the proof of Theorem 1.1.

Theorem 3.3. Assume that $M^{\prime} \cap \mathcal{K}=\{0\}$. For a given $X \in \mathcal{B}\left(\mathbb{H}^{2}\right)$ the following are true.

(i) The $X$ falls in $T\left(M^{\prime} \bar{\rtimes}_{\alpha} G\right)+\mathfrak{S}_{p}$ if and only if $X$ commutes with $T(\pi(M))$ modulo $\mathfrak{S}_{p}$ and satisfies that $T_{\lambda(s)}^{*} X T_{\lambda(s)}-X \in \mathfrak{S}_{p}$ for every $s \in S$.

(ii) The $X$ falls in $M^{\prime} \overline{\times}_{\alpha} S+\mathfrak{S}_{p}$ if and only if $X$ commutes with $T(\pi(M))$ and $\left\{T_{\lambda(s)} \mid s \in S\right\}$ modulo $\mathfrak{S}_{p}$.

Proof. First, we prove the 'if' part of (i). Let $X \in \operatorname{Esscom}_{p}(T(\pi(M)))$ be arbitrarily chosen in such a way that $T_{\lambda(s)}^{*} X T_{\lambda(s)}-X \in \mathfrak{S}_{p}$ for every $s \in S$. By Remark 2.2 there exist $a \in \pi(M)^{\prime}$ and $K \in \mathfrak{S}_{p}$ such that $X=T_{a}+K$. Note that $\left(T_{a}-\right.$ $\left.T_{\lambda(s)}^{*} T_{a} T_{\lambda(s)}\right) P=P\left(a-\lambda(s)^{*} a \lambda(s)\right) P \in \pi(M)^{\prime}$ since $a, P \in \pi(M)^{\prime}$ and $\lambda(s), s \in S$, normalize $\pi(M)^{\prime}$. For each $s \in S$ one has

$$
\left(T_{a}-T_{\lambda(s)}^{*} T_{a} T_{\lambda(s)}\right) P=\left(X-T_{\lambda(s)}^{*} X T_{\lambda(s)}\right) P-\left(K-T_{\lambda(s)}^{*} K T_{\lambda(s)}\right) P
$$

falls into $\mathcal{K} \cap \pi(M)^{\prime}$ by assumption. Thus, it follows from Lemma 3.1 that $T_{\lambda(s)}^{*} T_{a} T_{\lambda(s)}$ $=T_{a}$ for every $s \in S$, and hence we have $T_{a} \in T\left(M^{\prime} \bar{\rtimes}_{\alpha} G\right)$ by Proposition 2.6. Therefore, the $X=T_{a}+K$ is in $T\left(M^{\prime} \bar{\rtimes}_{\alpha} G\right)+\mathfrak{S}_{p}$. The 'only if' part of (i) follows from Proposition 2.6 again.

Next, we prove (ii). Since $M^{\prime} \overline{\times}_{\alpha} S=\left(S \overline{\times}_{\alpha} M\right)^{\prime}$, the 'only if' part is trivial. By the preceding paragraph, it suffices to prove that every $T_{y} \in T\left(M^{\prime} \bar{\rtimes}_{\alpha} G\right)$ commuting with $\left\{T_{\lambda(s)} \mid s \in S\right\}$ modulo $\mathfrak{S}_{p}$ falls in $M^{\prime} \overline{\times}_{\alpha} S=T\left(M^{\prime} \bar{\rtimes}_{\alpha} S\right)$. By Proposition 2.4 it suffices to show that $\left(1 \otimes e_{g}\right) y\left(1 \otimes e_{\iota}\right)=0$ for every $g \in G \backslash S$. Note that $\left(1 \otimes e_{g}\right) y\left(1 \otimes e_{\iota}\right) \in \pi(M)^{\prime}$ since $\left(1 \otimes e_{h}\right) \in \pi(M)^{\prime}$ for every $h \in G$. As in the proof of Proposition 2.6 we can find $s, t \in S$ in such a way that $g=s^{-1} t$, and have $\left\langle y \xi \otimes \delta_{\iota}, \eta \otimes \delta_{g}\right\rangle=\left\langle\left[T_{y}, T_{\lambda(s)}\right] \xi \otimes \delta_{\iota}, \eta \otimes \delta_{t}\right\rangle$ for every $\xi, \eta \in \mathcal{H}$, which implies that $\left(1 \otimes e_{g}\right) y\left(1 \otimes e_{\iota}\right)$ is compact, and hence $\left(1 \otimes e_{g}\right) y\left(1 \otimes e_{\iota}\right) \in \pi(M)^{\prime} \cap \mathcal{K}=\{0\}$. Therefore, we get $T_{y} \in M^{\prime} \overline{\times}_{\alpha} S$.

Proof of Corollary 1.2. By [4, Theorem 3.2] every action $\alpha: G \curvearrowright M$ is spatial. Since $M$ is anti-spatially isomorphic to $M^{\prime}$, it suffices to prove that $\mathcal{K} \cap M=\{0\}$. We first prove the case when $M=\mathcal{B}\left(\ell^{2}\right)$. Since $\left(\mathcal{B}\left(\ell^{2}\right), L^{2}\left(\mathcal{B}\left(\ell^{2}\right)\right)\right) \cong\left(\mathcal{B}\left(\ell^{2}\right) \otimes \mathbb{C} 1, \ell^{2} \otimes \overline{\ell^{2}}\right)$ and $\ell^{2}$ is infinite dimensional, we have $\mathcal{K} \cap\left(\mathcal{B}\left(\ell^{2}\right) \otimes \mathbb{C} 1\right)=\{0\}$. When $M$ is diffuse, it is clear that $\mathcal{K} \cap M=\{0\}$. The general case follows from [4, Lemma 2.6], which guarantees that each central projection $q \in M$ enjoys $\left(M q, q L^{2}(M)\right) \cong$ $\left(M q, L^{2}(M q)\right)$.

\section{Proof of Theorem 1.3}

Let $(M, \mathcal{H}, \alpha)$ be as in Theorem 1.3 . We first point out that the same assertions of the lemmas below hold true for $\rho(1)$ since $\lambda(1)$ and $\rho(1)$ are unitarily equivalent, see Remark 2.3. Remark that $\lambda(n)$ converge to 0 weakly, and hence $\lambda(n)^{*} K \lambda(n)$ converge to 0 strongly for every compact operator $K$. Also, note that $(1-P) \lambda(n)$ converges to 0 strongly. These facts are frequently used throughout.

The following lemma seems well-known, but we do give its proof for the sake of completeness.

Lemma 4.1. There exists a $*$-isomorphism from $L^{\infty}=L^{\infty}(\mathbb{T})$ onto $\{\lambda(1)\}^{\prime \prime}$ sending $z$ to $\lambda(1)$. 
Proof. Let $U$ be the bilateral shift on $L^{2}(\mathbb{T})$ with respect to the standard basis $\left\{z^{n} \mid n \in \mathbb{Z}\right\}$ and define the unitary transformation $V: \ell^{2}(\mathbb{Z}) \rightarrow L^{2}(\mathbb{T})$ by $V \delta_{n}:=$ $z^{n}, n \in \mathbb{Z}$. Then one has $\lambda(1)=1 \otimes \lambda_{1}=1 \otimes V^{*} U V$. Since the von Neumann algebra generated by $U$ is known to be $L^{\infty}$, the correspondence $L^{\infty} \ni f \mapsto 1 \otimes V^{*} f V \in$ $\{\lambda(1)\}^{\prime \prime}$ apparently gives the desired $*$-isomorphism.

Recall that the essential norm $\|X\|_{\mathrm{e}}$ of $X \in \mathcal{B}(\mathcal{H})$ is defined to be $\|X\|_{\mathrm{e}}=$ $\inf \{\|X-K\| \mid K \in \mathcal{K}(\mathcal{H})\}$ and that $X$ is said to be essentially normal if $X^{*} X-$ $X X^{*} \in \mathcal{K}(\mathcal{H})$.

Lemma 4.2. The operator $T_{\lambda(1)}$ is essentially normal and the image of $C^{*}\left(T_{\lambda(1)}\right)$ in the Calkin algebra is isomorphic to $C(\mathbb{T})$.

Proof. Since $T_{\lambda(1)}^{*} T_{\lambda(1)}-T_{\lambda(1)} T_{\lambda(1)}^{*}$ is the projection onto $\mathcal{H} \otimes \mathbb{C} \delta_{\iota}$ and $\mathcal{H}$ is finite dimensional, $T_{\lambda(1)}$ is essentially normal. For the second assertion it suffices to show that $\left\|T_{x}\right\|_{\mathrm{e}}=\|x\|$ for $x \in \mathbb{Z} \bar{\ltimes}_{\alpha} M$. Let $K \in \mathcal{K}\left(\mathbb{H}^{2}\right)$ be arbitrary. By [14, Poposition 3.4], $\rho(n)^{*} P x P \rho(n)$ converges to $x$ strongly. Since $K$ is compact, we have $x=\mathrm{s}-\lim _{n \rightarrow \infty} \rho(n)^{*}(P x P-K) \rho(n)$. By the lower-semicontinuity of operator norm, one has $\left\|T_{x}-K\right\| \geq\|x\|$. Since $K$ is arbitrary, we get $\left\|T_{x}\right\|_{\mathrm{e}}=\|x\|$.

Lemma 4.3. If $a \in \pi(M)^{\prime}$ satisfies that $\left[T_{a}, T_{\lambda(1)}\right] \in \mathcal{K}$, then every $\sigma$-weak cluster point of $\left\{\lambda(n)^{*} \operatorname{PaP} \lambda(n)\right\}_{n \geq 0}$ falls in $M^{\prime} \bar{\rtimes}_{\alpha} \mathbb{Z}$.

Proof. Let $b$ be a $\sigma$-weak cluster point of $\left\{\lambda(n)^{*} P a P \lambda(n)\right\}_{n \geq 0}$. Then there exists a subnet $\Lambda$ of $\mathbb{N}$ such that $b=\sigma-\mathrm{w}-\lim _{n \in \Lambda} \lambda(n)^{*} P a P \lambda(n)$. Since $P a P \in \pi(M)^{\prime}$ and $\lambda(1)$ normalizes $\pi(M)^{\prime}$, one has $b \in \pi(M)^{\prime}$. Hence, it suffices to show that the $b$ commutes with $\lambda(1)$. Since $[P a P, P \lambda(1) P]$ is compact by assumption, one has

$$
\begin{aligned}
& \lambda(1)\left(\lambda(n)^{*} \operatorname{PaP} \lambda(n)\right) \\
& =\lambda(n)^{*} P \lambda(1) P a P \lambda(n) \\
& =\lambda(n)^{*} P a P \lambda(1) P \lambda(n)+\lambda(n)^{*}[P \lambda(1) P, P a P] \lambda(n) \\
& =\lambda(n)^{*} P a P \lambda(n) \lambda(1)+\lambda(n)^{*}[P \lambda(1) P, P a P] \lambda(n)+\lambda(n)^{*} P a P \lambda(1)(1-P) \lambda(n) \\
& \stackrel{n \in \Lambda}{\longrightarrow} b \lambda(1) \quad \text { strongly, }
\end{aligned}
$$

which implies $[b, \lambda(1)]=0$.

We denote by $\|X\|_{p} \in[0,+\infty]$ the Schatten $p$-norm of $X \in \mathcal{B}(\mathcal{H})$ with $1 \leq p<\infty$ and define $\|X\|_{\infty}:=\|X\|$, the operator norm of $X$. Recall the fact that the norms $\|\cdot\|_{p}$ are lower-semicontinuous with respect to the weak operator topology (see e.g. [6. Proposition 2.11]).

Lemma 4.4. Let $b \in\{\lambda(1)\}^{\prime}, K \in \mathcal{K}$, and $1 \leq p \leq \infty$ be given and set $X:=T_{b}+K$. Assume that $x$ is an element in the *-algebra generated by $\lambda(1)$ and there exists a constant $\delta>0$ such that $\left\|T_{x} X-T_{b x}\right\|_{p}>\delta$. Then $\left\|\left[T_{x \lambda(n)}, X\right]\right\|_{p}>\delta$ and $x \lambda(n) \in \mathbb{Z}_{+} \bar{\aleph}_{\alpha} M$ hold for all sufficiently large $n \in \mathbb{N}$.

Proof. Since $x$ is a polynomial of $\lambda(1)$ and $\lambda(1)^{*}$, there exists $n_{0} \in \mathbb{N}$ such that $x \lambda\left(n_{0}\right)$ is in $\mathbb{Z}_{+} \bar{\ltimes}_{\alpha} M$. For $n \geq n_{0}$ one has

$$
\begin{aligned}
T_{\lambda(n)}^{*}\left[T_{x} T_{\lambda(n)}, X\right] & =T_{\lambda(n)}^{*} T_{x} T_{\lambda(n)} X-T_{\lambda(n)}^{*}\left(T_{b}+K\right) T_{x} T_{\lambda(n)} \\
& =T_{x} X-T_{\lambda(n) b}^{*} T_{x \lambda(n)}-T_{\lambda(n)}^{*} K T_{x} T_{\lambda(n)} \\
& =T_{x} X-T_{b x}-T_{\lambda(n)}^{*} K T_{x} T_{\lambda(n)}
\end{aligned}
$$


K. HASEGAWA

converges to $T_{x} X-T_{b x}$ strongly as $n \rightarrow \infty$, since $K$ is compact. Thus, by the lower-semicontinuity of $\|\cdot\|_{p}$, there exists $n_{1}>n_{0}$ such that

$$
\left\|\left[T_{x \lambda(n)}, X\right]\right\|_{p} \geq\left\|T_{\lambda(n)}^{*}\left[T_{x} T_{\lambda(n)}, X\right]\right\|_{p}>\delta
$$

as long as $n \geq n_{1}$.

First, we deal with the case of $p=\infty$. Although Claim 4.5 below can be shown in the same way as the proof of [2, Theorem 2], we do give its a bit simplified proof based on the techniques used in 12 and [16. The way of our proof may be essentially known, but we could not find a suitable reference that explicitly explains such an argument.

Proof of Theorem 1.3 when $p=\infty$. Let $X$ be in $\operatorname{Esscom}_{\infty}\left(\mathbb{Z}_{+} \bar{x}_{\alpha} M\right)$. Since $X$ is in $\operatorname{Esscom}_{\infty}(T(\pi(M)))=T\left(\pi(M)^{\prime}\right)+\mathcal{K}$, there exists $a \in \pi(M)^{\prime}$ such that $X-T_{a}$ is compact. Let $\Lambda$ be a subsequence of $\mathbb{N}$ such that the limit $b=\sigma$-w$\lim _{n \in \Lambda} \lambda(n)^{*} \operatorname{PaP} \lambda(n)$ exists. Lemma 4.3 says that this $b$ must be in $M^{\prime} \bar{\rtimes}_{\alpha} \mathbb{Z}$. If $T_{a}-T_{b}$ is compact, then so is $X-T_{b}$ too. Since $T_{a}$ is in $\operatorname{Esscom}_{\infty}\left(\mathbb{Z}_{+} \bar{x}_{\alpha} M\right)$, that is, $[X, Y]$ is compact for every $Y \in \mathbb{Z}_{+} \bar{x}_{\alpha} M$, the proof will be complete after establishing the next claim.

Claim 4.5. If $T_{a}-T_{b}$ is not compact, then there exists $Z$ in $\mathbb{Z}_{+} \bar{\aleph}_{\alpha} M$ such that $\left[T_{Z}, T_{a}\right]$ is not compact.

$(\because)$. Set $\delta:=\left\|T_{a}-T_{b}\right\|_{\mathrm{e}}>0$. By Lemma 4.2 the image of $C^{*}\left(T_{\lambda(1)}\right)$ in the Calkin algebra is isomorphic to $C(\mathbb{T})$. As in the proof of [16, Proposition 8] we can choose $\gamma \in \mathbb{T}$ in such a way that $f(\gamma)=1$ forces

$$
\left\|T_{\hat{f}} T_{a}-T_{\hat{f} b}\right\|_{\mathrm{e}}=\left\|T_{\hat{f}}\left(T_{a}-T_{b}\right)\right\|_{\mathrm{e}}>\delta / 2
$$

for $f \in C(\mathbb{T})$, where $L^{\infty} \ni g \mapsto \hat{g} \in\{\lambda(1)\}^{\prime \prime}$ is the $*$-isomorphism in Lemma 4.1] Let us construct a sequence $\left\{p_{k}\right\}_{k} \subset \mathbb{Z}_{+} \bar{\ltimes}_{\alpha} M$ such that $\lim _{k \rightarrow \infty}\left\|\left[T_{p_{k}}, T_{a}\right]\right\| \geq \delta / 2$ and the sum $\sum_{k=1}^{\infty} p_{k}$ converges strongly. Set $B_{n}^{\gamma}:=\left\{\gamma^{\prime} \in \mathbb{T}|| \gamma^{\prime}-\gamma \mid<n^{-1}\right\}$ and $c(f):=\left\|T_{\hat{f}} T_{a}-T_{\hat{f} b}\right\|$ for $f \in L^{\infty}(\mathbb{T})$. Note that $c(\cdot)$ is $\sigma$-weakly lowersemicontinuous. For $n+2<k$ one can take $f_{n, k} \in C(\mathbb{T})$ in such a way that $0 \leq f_{n, k} \leq 1, f=0$ on $B_{k}^{\gamma} \cup\left(\mathbb{T} \backslash B_{n}^{\gamma}\right)$ and $f=1$ on $B_{n+1}^{\gamma} \backslash B_{k-1}^{\gamma}$. Note that $\left\{f_{n, k}\right\}_{k}$ converges almost everywhere to a function $f_{n} \in C(\mathbb{T})$ with $f_{n}(\gamma)=1$. Hence, by the lower-semicontinuity of $c(\cdot)$ together with Lebesgue's dominated convergence theorem, there exists $n^{\prime}>n$ such that $c\left(f_{n, n^{\prime}}\right)>\delta / 2$. We can inductively choose $n_{1}<n_{1}^{\prime}<n_{2}<n_{2}^{\prime}<\ldots$ in such a way that $g_{k}:=f_{n_{k}, n_{k}^{\prime}}$ satisfies that $0 \leq g_{k} \leq 1$, $\left\{g_{k}\right\}_{k}$ have pairwise disjoint supports, and $c\left(g_{k}\right)>\delta / 2$. Let $h_{k}$ be a trigonometric polynomial such that $\left\|g_{k}-h_{k}\right\|_{\infty}<2^{-k}$ and $c\left(h_{k}\right)>\delta / 2$. By Lemma 4.4 one can choose $m_{k}$ such that $\left\|\left[T_{\hat{h_{k}} \lambda\left(m_{k}\right)}, T_{a}\right]\right\|>\delta / 2$ and $\hat{h_{k}} \lambda\left(m_{k}\right) \in \mathbb{Z}_{+} \bar{\ltimes}_{\alpha} M$. Set $p_{k}:=\hat{h_{k}} \lambda\left(m_{k}\right)$, then $\left\|\left[T_{p_{k}}, T_{a}\right]\right\| \leq 2\left\|h_{k}\right\|_{\infty}\|a\| \leq 4\|a\|$. Thus by passing to a subsequence if necessary, we may and do assume that $\lim _{k \rightarrow \infty}\left\|\left[T_{p_{k}}, T_{a}\right]\right\|$ exists. Let $\chi_{k}$ be the characteristic function of $\operatorname{supp}\left(g_{k}\right)$. Then $\hat{\chi_{k}}, k \in \mathbb{N}$, are mutually orthogonal projections in $\{\lambda(1)\}^{\prime \prime}$ satisfying that $\left\|p_{k}\left(1-\hat{\chi_{k}}\right)\right\|=\left\|h_{k}\left(1-\chi_{k}\right)\right\|_{\infty}<$ $2^{-k}$ and $\left\|p_{k} \hat{\chi_{k}}\right\|=\left\|\hat{\chi_{k}} p_{k} \hat{\chi_{k}}\right\| \leq 2$ for every $k \in \mathbb{N}$. For any $\xi \in \mathbb{L}^{2}$ and $k<l$ we have

$$
\left\|\sum_{i=k}^{l} p_{i} \xi\right\| \leq\left\|\sum_{i=k}^{l} \hat{\chi}_{i} p_{i} \hat{\chi}_{i} \xi\right\|+\left\|\sum_{i=k}^{l} p_{i}\left(1-\hat{\chi}_{i}\right) \xi\right\| \leq 2\left\|\sum_{i=k}^{l} \hat{\chi}_{i} \xi\right\|+2^{-(k-1)}\|\xi\|
$$


converges to 0 as $k, l \rightarrow \infty$, and hence the $\left\{p_{k}\right\}_{k}$ is the desired sequence. Note that $p_{k}$ and $p_{k}^{*}$ converge to 0 strongly. Since $T_{a} \in \operatorname{Esscom}_{\infty}\left(\mathbb{Z}_{+} \bar{x}_{\alpha} M\right)$, we can apply [12, Lemma 2.1] to compact operators $\left[T_{p_{k}}, T_{a}\right]$, and obtain a subsequence $\left\{p_{k(i)}\right\}_{i=1}^{\infty}$ such that

$$
\sum_{i=1}^{\infty}\left[T_{p_{k(i)}}, T_{a}\right] \text { converges strongly and }\left\|\sum_{i=1}^{\infty}\left[T_{p_{k(i)}}, T_{a}\right]\right\|_{\mathrm{e}} \geq \delta / 2 .
$$

Letting $Z:=\sum_{i=1}^{\infty} p_{k(i)}$ we have $Z \in \mathbb{Z}_{+} \bar{\ltimes}_{\alpha} M$ and $\left[T_{Z}, T_{a}\right] \notin \mathcal{K}$, which implies the claim.

We then treat the case of $p \neq \infty$. The next lemma originates in [12, Lemma 2.1].

Lemma 4.6. Let $\mathcal{H}_{1}$ be a Hilbert spaces and fix $1 \leq p<\infty$. Assume that a sequence $\left\{K_{n}\right\}_{n} \subset \mathfrak{S}_{p}\left(\mathcal{H}_{1}\right)$ satisfies the following conditions:

(A1) $\left\|K_{n}\right\|_{p}>2$ for every $n \in \mathbb{N}$.

(A2) $\sup _{n}\left\|K_{n}\right\|<C_{1}$ for some $C_{1}>0$.

(A3) $K_{n}$ and $K_{n}^{*}$ converge to 0 strongly.

Then there exists a subsequence $\left\{K_{n_{k}}\right\}_{k=1}^{\infty}$ such that $\sum_{k=1}^{\infty} K_{n_{k}}$ converges strongly and $\sum_{k=1}^{\infty} K_{n_{k}} \notin \mathfrak{S}_{p}\left(\mathcal{H}_{1}\right)$.

Proof. Let $\mathcal{H}_{0}$ be the separable Hilbert space generated by $\bigcup_{n=1}^{\infty}\left(\operatorname{Ker} K_{n}\right)^{\perp}$. Choose a CONS $\left\{e_{n}\right\}_{n=1}^{\infty}$ of $\mathcal{H}_{0}$, and let $R_{n}$ be the orthogonal projection onto the linear span of $e_{1}, \ldots, e_{n}$. We claim that there exist mutually orthogonal finite rank projections $\left\{Q_{k}\right\}_{k=1}^{\infty}$ and a subsequence $\left\{K_{n_{k}}\right\}_{k=1}^{\infty}$ such that

(1) $\left\|Q_{k} K_{n_{k}} Q_{k}\right\|_{p}>1$,

(2) $\left\|K_{n_{k}} Q_{k}^{\perp}\right\|_{p}<3^{-k}$ and $\left\|Q_{k}^{\perp} K_{n_{k}}\right\|_{p}<3^{-k}$,

(3) $\left\|K_{n_{k}} R_{k}\right\|<2^{-k}$,

with $Q_{k}^{\perp}:=I-Q_{k}$. Assume that we have chosen $Q_{1}, \ldots, Q_{k}$ and $n_{1}, \ldots, n_{k}$. Put $Q:=\sum_{j=1}^{k} Q_{j}$. Since $K_{n}, K_{n}^{*} \rightarrow 0$ strongly and $Q$ is finite rank, there exists $n_{k+1}>$ $n_{k}$ such that $\left\|K_{n_{k+1}} R_{k+1}\right\|<2^{-k-1},\left\|K_{n_{k+1}} Q\right\|_{p}<3^{-k-1}$, and $\left\|Q K_{n_{k+1}}\right\|_{p}<$ $3^{-k-1}$. Thus this $K_{n_{k+1}}$ satisfies the desired (3). Write $K:=K_{n_{k+1}}$ for short. We have

$$
2<\|K\|_{p} \leq\left\|Q^{\perp} K Q^{\perp}\right\|_{p}+\left\|Q K Q^{\perp}\right\|_{p}+\|K Q\|_{p}<\left\|Q^{\perp} K Q^{\perp}\right\|_{p}+1,
$$

implying $\left\|Q^{\perp} K Q^{\perp}\right\|_{p}>1$. Let $F_{j} \leq Q^{\perp}, j \in J$, be an increasing net of finite rank projections which converges to $Q^{\perp}$ strongly. Since $K$ is in $\mathfrak{S}_{p}$, one has $\| F_{i} K F_{i}-$ $Q^{\perp} K Q^{\perp}\left\|_{p},\right\| K F_{i}^{\perp} \|_{p}$, and $\left\|F_{i}^{\perp} K\right\|_{p}$ converge to 0 . By the lower-semicontinuity of norm, we can find $j \in J$ in such a way that $Q_{k+1}:=F_{j}<Q^{\perp}$ satisfies (1) and (2) for $K=K_{n_{k+1}}$. Hence we can construct the desired $Q_{k}$ and $K_{n_{k}}$ by induction.

Write $K_{k}:=K_{n_{k}}$ for short. By (A2) and (2) we have

$$
\left\|\sum_{k=1}^{n} K_{k}\right\| \leq\left\|\sum_{k=1}^{n} Q_{k} K_{k} Q_{k}\right\|+\sum_{k=1}^{n}\left\|Q_{k}^{\perp} K_{k} Q_{k}\right\|+\sum_{k=1}^{n}\left\|K_{k} Q_{k}^{\perp}\right\|<C_{1}+2 .
$$


K. HASEGAWA

Hence $\sum_{k=1}^{n} K_{k}$ is norm bounded. If $\xi$ in $R_{n} \mathcal{H}_{0}$ and $m \geq l \geq n$, then by (3) we have

$$
\left\|\left(\sum_{k=1}^{m} K_{k}-\sum_{k=1}^{l} K_{k}\right) \xi\right\| \leq \sum_{k=l+1}^{m}\left\|K_{k} R_{n}\right\|\|\xi\| \leq \sum_{k=l+1}^{m} 2^{-k}\|\xi\| \leq 2^{-l}\|\xi\| .
$$

Since $\bigcup_{n=1}^{\infty} R_{n} \mathcal{H}_{1}$ is dense in $\mathcal{H}_{0}$ and each $K_{k}$ equals to 0 on $\mathcal{H}_{0}^{\perp}, \sum_{k=1}^{\infty} K_{k}$ converges strongly. Set $X=\sum_{k=1}^{\infty} K_{k}$. Since each $Q_{n}$ is finite rank, $Q_{n} \sum_{k=1}^{m} K_{k} Q_{n}$ converges to $Q_{n} X Q_{n}$ in norm. For each $n \in \mathbb{N}$ we have,

$$
\begin{aligned}
\left\|Q_{n} X Q_{n}\right\|_{p} & =\lim _{m \rightarrow \infty}\left\|Q_{n} \sum_{k=1}^{m} K_{k} Q_{n}\right\|_{p} \geq\left\|Q_{n} K_{n} Q_{n}\right\|_{p}-\sum_{k \neq n}\left\|Q_{n} K_{k} Q_{n}\right\|_{p} \\
& \geq 1-\sum_{k \neq n}\left\|K_{k}\left(I-Q_{k}\right)\right\|_{p} \geq 1-\sum_{k \neq n} 3^{-k} \geq \frac{1}{2},
\end{aligned}
$$

which implies that $X \notin \mathfrak{S}_{p}$, since $Q_{n}, n \in \mathbb{N}$, are mutually orthogonal.

We can now complete the proof of Theorem 1.3 .

Proof of Theorem 1.3 when $p \neq \infty$. Let $X \in \operatorname{Esscom}_{p}\left(\mathbb{Z}_{+} \bar{x}_{\alpha} M\right)$ be arbitrarily chosen. Since $\operatorname{Esscom}_{p}\left(\mathbb{Z}_{+} \bar{x}_{\alpha} M\right) \subset \operatorname{Esscom}_{\infty}\left(\mathbb{Z}_{+} \bar{x}_{\alpha} M\right)$, there exists $b \in M^{\prime} \bar{\rtimes}_{\alpha} \mathbb{Z}$ such that $X-T_{b}$ is compact. Suppose that $X-T_{b} \in \mathcal{K} \backslash \mathfrak{S}_{p}$. Define $c_{p}: L^{\infty} \rightarrow[0,+\infty]$ by $c_{p}(g)=\|T(\hat{g}) X-T(b \hat{g})\|_{p}$. Note that $c_{p}$ is lower-semicontinuous with respect to the weak operator topology and $c_{p}(1)=+\infty$. Suppose that for each $\gamma \in \mathbb{T}$ there exists $n \in \mathbb{N}$ such that $c_{p}(f) \leq 2$ as long as $f \in C(\mathbb{T})$ satisfies that $0 \leq f \leq 1$ and $\operatorname{supp}(f) \subset B_{n}^{\gamma}$. Then, by the compactness of $\mathbb{T}$, there exist such $\left(\gamma_{1}, n_{1}\right), \ldots,\left(\gamma_{k}, n_{k}\right) \in \mathbb{T} \times \mathbb{N}$ with $\mathbb{T}=\bigcup_{i=1}^{k} B_{n_{i}}^{\gamma_{i}}$. Taking a partition of the unity $\left\{\psi_{i}\right\}_{i=1}^{k}$ for the covering $\left\{B_{n_{i}}^{\gamma_{i}}\right\}_{i=1}^{k}$, we have $c_{p}(1) \leq \sum_{i=1}^{k} c_{p}\left(\psi_{i}\right) \leq 2 k$, a contradiction. Hence, we can find $\gamma \in \mathbb{T}$ such that for every $n \in \mathbb{N}$ there exists $f_{n} \in C(\mathbb{T})$ such that $c_{p}\left(f_{n}\right)>2,0 \leq f_{n} \leq 1$, and $\operatorname{supp}\left(f_{n}\right) \subset B_{n}^{\gamma}$. By a same approximation argument as in the case of $p=\infty$ together with Lemma 4.4. we obtain $p_{k} \in \mathbb{Z}_{+} \bar{\ltimes}_{\alpha} M$ such that $p_{k}$ and $p_{k}^{*}$ converge to 0 strongly, $\sum_{k=1}^{\infty} p_{k}$ converges strongly, and that $\left\|p_{k}\right\| \leq 2$ and $\left\|\left[T_{p_{k}}, X\right]\right\|_{p}>2$ for every $k \in \mathbb{N}$. Applying Lemma 4.6 to $K_{k}=\left[T_{p_{k}}, X\right]$ we obtain a subsequence $\left\{p_{k(i)}\right\}_{i}$ such that $Z:=\sum_{i=1}^{\infty} p_{k(i)} \in \mathbb{Z}_{+} \bar{\ltimes}_{\alpha} M$ and $\left[T_{Z}, X\right] \notin \mathfrak{S}_{p}$. This is a contradiction; hence $X-T_{b}$ is in $\mathfrak{S}_{p}$.

Remark 4.7. To compute the essential fixed-points, there is a technical obstruction: In the proof of Lemma 4.3, it is crucial that that $\lambda(1)$ normalizes $\pi(M)^{\prime}$. In [16] Xia computed Essfix $x_{\infty}\left(T\left(H^{\infty}\right)\right)$ by using the finite Blaschke product $w_{n}$ instead of the inner function $z^{n}$, see [16, Proposition 3]. However, unitary elements in $C^{*}(\lambda(1))$ do not normalize $\pi(M)^{\prime}$ in general.

\section{The Condition $(\star)$}

Let $M$ be a von Neumann algebra on $L^{2}(M)$ and $\alpha$ be a $*$-automorphism of $M$. We say $(M, \alpha)$ satisfies the condition $(\star)$ if

$$
\operatorname{Esscom}_{p}\left(\mathbb{Z}_{+} \bar{x}_{\alpha} M\right) \subset T\left(M^{\prime} \bar{\rtimes}_{\alpha} \mathbb{Z}\right)+\mathfrak{S}_{p}, \quad 1 \leq p \leq \infty
$$

hold true. Note that $(M, \alpha)$ satisfies $(\star)$ if and only if so does $\left(M^{\prime}, \alpha\right)$ if and only if

$$
\operatorname{Esscom}_{p}\left(M \overline{\times}_{\alpha} \mathbb{Z}_{+}\right) \subset T\left(\mathbb{Z} \bar{\ltimes}_{\alpha} M^{\prime}\right)+\mathfrak{S}_{p}, \quad 1 \leq p \leq \infty
$$


hold. We have already seen that $(M, \alpha)$ satisfies $(\star)$ when $M$ is either diffuse, type $\mathrm{I}_{\infty}$ factor, a direct sum of them (Corollary 1.2), or finite dimensional (Theorem 1.3). In fact, we can prove the next theorem which asserts the condition $(\star)$ is satisfied in a more general case. For a given $(M, \alpha)$ it is known and not hard to see that $M$ is decomposed uniquely as $M=M_{\mathrm{c}} \oplus M_{\infty} \oplus \sum_{n \geq 1}^{\oplus} M_{n}$, where $M_{\mathrm{c}}$ is diffuse, the $M_{\infty}$ a direct sum of infinite type I factors, and $M_{n}=M_{n}(\mathbb{C}) \otimes \ell^{\infty}\left(\mathfrak{X}_{n}\right), n \geq 1$, with discrete sets $\mathfrak{X}_{n}$. The uniqueness of this decomposition guarantees that $\alpha$ is also decomposed as $\alpha=\alpha_{\mathrm{c}} \oplus \alpha_{\infty} \oplus \sum_{n \geq 1}^{\oplus} \alpha_{n}$. It is not difficult to see that there exists a unique automorphism $\beta_{n}$ of $\ell^{\infty}\left(\mathfrak{X}_{n}\right)$ and a unitary element $v_{n} \in M_{n}(\mathbb{C}) \otimes \ell^{\infty}\left(\mathfrak{X}_{n}\right)$ such that $\alpha_{n}=\operatorname{Ad} v_{n} \circ\left(\right.$ id $\left.\otimes \beta_{n}\right)$. Remark that $\beta_{n}$ induces a unique bijection $\theta_{n}$ on $\mathfrak{X}_{n}$. With these notation we have:

Theorem 5.1. Assume that every orbit of $\theta_{n}$ forms a finite set. Then $(M, \alpha)$ satisfies the condition $(\star)$.

To prove this theorem we need the following lemma.

Lemma 5.2. Let $\left\{\left(M_{i}, \alpha_{i}\right)\right\}_{i \in I}$ be a family of von Neumann algebras and their *-automorphisms. Set $M:=\sum_{i \in I}^{\oplus} M_{i}$ and $\alpha:=\sum_{i \in I}^{\oplus} \alpha_{i}$. If every $\left(M_{i}, \alpha_{i}\right)$ satisfies the condition $(\star)$, then so does $(M, \alpha)$.

Proof. Let $X \in \operatorname{Esscom}_{p}\left(\mathbb{Z}_{+} \bar{x}_{\alpha} M\right)$ be arbitrarily chosen. Let $e_{i}$ be the central support of $\pi\left(M_{i}\right)$ in $\pi(M)$ and put $A:=\left\{e_{i} \mid i \in I\right\}^{\prime \prime}$. Note that $\lambda(1)$ and $P$ commute with $A$. Then there exists $a \in \pi(M)^{\prime} \subset A^{\prime}$ such that $X-T_{a} \in \mathfrak{S}_{p}$. Since $T_{a}$ is also in $\operatorname{Esscom}_{p}\left(\mathbb{Z}_{+} \bar{x}_{\alpha} M\right)$ and $\mathbb{Z}_{+} \bar{x}_{\alpha} M=\sum_{i \in I}^{\oplus} \mathbb{Z}_{+} \bar{x}_{\alpha_{i}} M_{i}$, we have $T_{a e_{i}} \in \operatorname{Esscom}_{p}\left(\mathbb{Z}_{+} \bar{x}_{\alpha_{i}} M_{i}\right)$. By assumption, there exist $b_{i} \in M_{i}^{\prime} \bar{\rtimes}_{\alpha_{i}} \mathbb{Z}$ and $K_{i} \in$ $\mathfrak{S}_{p}\left(e_{i} L^{2}(M)\right)$ such that $T_{a e_{i}}=T_{b_{i}}+K_{i}$. Set $b:=\sum_{i \in I} b_{i}$ and $K:=\sum_{i \in I} K_{i}$. Since $b \in \sum_{i \in I}^{\oplus} M_{i}^{\prime} \bar{\rtimes}_{\alpha_{i}} \mathbb{Z}=M^{\prime} \bar{\rtimes}_{\alpha} \mathbb{Z}$, it suffices to prove that $K \in \mathfrak{S}_{p}$. Since $T_{a e_{i}}-T_{\lambda(n) e_{i}}^{*} T_{a e_{i}} T_{\lambda(n) e_{i}} \rightarrow K_{i}$ strongly as $n \rightarrow \infty$, the lower-semicontinuity of $\|\cdot\|_{p}$ enable us to find $n_{i} \in \mathbb{N}$ in such a way that

$$
\left\|\left[T_{\lambda\left(n_{i}\right) e_{i}}, T_{a e_{i}}\right]\right\|_{p} \geq\left\|T_{a e_{i}}-T_{\lambda\left(n_{i}\right) e_{i}}^{*} T_{a e_{i}} T_{\lambda\left(n_{i}\right) e_{i}}\right\|_{p}>2^{-1}\left\|K_{i}\right\|_{p} .
$$

Then $x:=\sum_{i \in I} \lambda\left(n_{i}\right) e_{i}$ falls in $\mathbb{Z}_{+} \bar{\ltimes}_{\alpha} M$. Since $T_{a} \in \operatorname{Esscom}_{p}\left(\mathbb{Z}_{+} \overline{\times}_{\alpha} M\right)$, one has $\left[T_{x}, T_{a}\right]=\sum_{i \in I}\left[T_{\lambda\left(n_{i}\right) e_{i}}, T_{a e_{i}}\right] \in \mathfrak{S}_{p}$. Hence, it follows from the inequality above that $K \in \mathfrak{S}_{p}$.

Proof of Theorem 5.1. By Corollary 1.2 and Lemma 5.2 we may and do assume that $M=M_{n}$ and $\alpha=$ id $\otimes \beta_{n}$. Decompose $\mathfrak{X}_{n}$ into the disjoint $\theta_{n}$-orbits $\mathfrak{X}_{n, j}$, $j \in J_{n}$. Set $M_{n, j}:=M_{n}(\mathbb{C}) \otimes \ell^{\infty}\left(\mathfrak{X}_{n, j}\right)$, which sits inside $M_{n}=M_{n}(\mathbb{C}) \otimes \ell^{\infty}\left(\mathfrak{X}_{n}\right)$ naturally. Then $M_{n}=\sum_{j \in J_{n}}^{\oplus} M_{n, j}$, and clearly $\alpha\left(M_{n, j}\right)=M_{n, j}$ holds for every $j \in J_{n}$. Consequently, one has $\left(M_{n}, \alpha_{n}\right)=\sum_{j \in J_{n}}^{\oplus}\left(M_{n, j},\left.\alpha\right|_{M_{n, j}}\right)$. By assumption each $\mathfrak{X}_{n, j}$ is a finite set, and hence $M_{n, j}$ is finite dimensional. Therefore, the desired assertion follows from Theorem 1.3 thanks to Lemma 5.2 .

Here a question naturally arises.

Question 5.3. Let $\sigma$ be the $*$-automorphism on $\ell^{\infty}(\mathbb{Z})$ induced from the translation $n \in \mathbb{Z} \mapsto n+1 \in \mathbb{Z}$. Does $\left(\ell^{\infty}(\mathbb{Z}), \sigma\right)$ satisfy the condition $(\star)$ ?

In fact, the positive answer to the question enables us to get rid of the assumption from Theorem 5.1 as follows. We use the notation in the proof of Theorem 5.1 Thanks to Lemma 5.2 and Theorem [5.1, it suffices to prove that for every infinite 
$\theta_{n}$-orbit $\mathfrak{X}_{n, j},\left(M_{n, j},\left.\alpha\right|_{M_{n, j}}\right)$ satisfies the condition $(\star)$. Then $\left(M_{n, j},\left.\alpha\right|_{M_{n, j}}\right)$ can be identified with $\left(M_{n}(\mathbb{C}) \otimes \ell^{\infty}(\mathbb{Z}), \operatorname{Ad} v_{n, j} \circ\right.$ id $\left.\otimes \sigma\right)$ for a unitary element $v_{n, j} \in$ $M_{n}(\mathbb{C}) \otimes \ell^{\infty}\left(\mathfrak{X}_{n, j}\right)$, and hence we may assume that $(M, \alpha)=\left(M_{n}(\mathbb{C}) \otimes \ell^{\infty}(\mathbb{Z})\right.$, id $\left.\otimes \sigma\right)$. Write $\ell^{\infty}:=\ell^{\infty}(\mathbb{Z})$ and $\ell^{2}:=\ell^{2}(\mathbb{Z})$ for simplicity. The standard form of $M_{n}(\mathbb{C}) \otimes \ell^{\infty}$ becomes $M_{n}(\mathbb{C}) \otimes \mathbb{C} 1 \otimes \ell^{\infty}$ on $\mathbb{C}^{n} \otimes \mathbb{C}^{n} \otimes \ell^{2}$. Hence, $\mathbb{Z} \bar{\ltimes}_{\text {id } \otimes \sigma}\left(M_{n}(\mathbb{C}) \otimes \ell^{\infty}\right)$ and $\left(M_{n}(\mathbb{C}) \otimes \ell^{\infty}\right)^{\prime} \bar{\rtimes}_{\mathrm{id} \otimes \sigma} \mathbb{Z}$ become $M_{n}(\mathbb{C}) \otimes \mathbb{C} 1 \otimes\left(\mathbb{Z} \bar{\ltimes}_{\sigma} \ell^{\infty}\right)$ and $\mathbb{C} 1 \otimes M_{n}(\mathbb{C}) \otimes\left(\ell^{\infty} \bar{\rtimes}_{\sigma} \mathbb{Z}\right)$, respectively. It is easily seen that

$$
\operatorname{Esscom}_{p}\left(M_{n}(\mathbb{C}) \otimes \mathbb{C} 1 \otimes\left(\mathbb{Z}_{+} \bar{x}_{\sigma} \ell^{\infty}\right)\right) \subset \mathbb{C} 1 \otimes M_{n}(\mathbb{C}) \otimes \operatorname{Esscom}_{p}\left(\mathbb{Z}_{+} \bar{x}_{\sigma} \ell^{\infty}\right)+\mathfrak{S}_{p} .
$$

Therefore, if Question 5.3 had an affirmative answer, then $(M, \alpha)$ would satisfy the condition $(\star)$ since

$$
\begin{aligned}
\operatorname{Esscom}_{p}\left(\mathbb{Z}_{+} \bar{x}_{\alpha} M\right) & =\operatorname{Esscom}_{p}\left(M_{n}(\mathbb{C}) \otimes \mathbb{C} 1 \otimes\left(\mathbb{Z}_{+} \bar{x}_{\sigma} \ell^{\infty}\right)\right) \\
& \subset \mathbb{C} 1 \otimes M_{n}(\mathbb{C}) \otimes \operatorname{Esscom}_{p}\left(\mathbb{Z}_{+} \bar{x}_{\sigma} \ell^{\infty}\right)+\mathfrak{S}_{p} \\
& \subset \mathbb{C} 1 \otimes M_{n}(\mathbb{C}) \otimes T\left(\ell^{\infty} \bar{\rtimes}_{\sigma} \mathbb{Z}\right)+\mathfrak{S}_{p} \\
& =T\left(M^{\prime} \bar{\rtimes}_{\alpha} \mathbb{Z}\right)+\mathfrak{S}_{p} .
\end{aligned}
$$

Finally, we should remark that the canonical implementing unitary operator of $\sigma$ is nothing but the bilateral shift on $\ell^{2}(\mathbb{Z})$ with respect to the standard basis. Hence Question 5.3 seems operator theoretic rather than operator algebraic.

\section{ACKNOWLEDGMENT}

The author wishes to express his sincere gratitude to his supervisor, Professor Yoshimichi Ueda for his passionate guidance and continuous encouragement.

\section{REFERENCES}

[1] A. Brown and P.R. Halmos, Algebraic properties of Toeplitz operators. J. Reine Angew. Math. 213 1963/1964 89-102.

[2] K.R. Davidson, On operators commuting with Toeplitz operators modulo the compact operators, J. Funct. Anal. 24 (1977) 291-302.

[3] R.G. Douglas, Banach Algebra Techniques in Operator Theory, second ed., Grad. Texts in Math. 179, Springer-Verlag, New York, 1998.

[4] U. Haagerup, The standard form of von Neumann algebras, Math. Scand. 37 (1975), no. 2, $271-283$.

[5] E. Hewiit and K. A. Ross, Abstract Harmonic Analysis, Vol. I, second ed., Grundlehren der Mathematischen Wissensrhaften [Fundamental Principles of Mathematical Sciences], vol. 115, Springer-Verlag, Berlin, 1979.

[6] F. Hiai, Log-majorizations and norm inequalities for exponential operators, in Linear Operators. Banach Center Publ, 38, 119-181 (1997)

[7] T.S. Hoover, Derivations, homomorphisms, and operator ideals, Proc. Amer. Math. Soc. 62 (1977), no. 2, 293-298.

[8] B.E. Johnson and S.K. Parrott, Operators commuting with a von Neumann algebra modulo the set of compact operators, J. Funct. Anal. 11 (1972), 39-61.

[9] E. Kakariadis, Semicrossed products and reflexivity. J. Operator Theory 67 (2012), no. 2, 379-395.

[10] R.I. Loebl and P.S. Muhly, Analyticity and flows in von Neumann algebras. J. Funct. Anal. 29 (1978), no. 2, 214-252.

[11] M. McAsey, P.S. Muhly, and K-S. Saito, Nonselfadjoint crossed products (invariant subspaces and maximality). Trans. Amer. Math. Soc. 248 (1979), no. 2, 381-409.

[12] P.S. Muhly and J. Xia, On automorphisms of the Toeplitz algebra, Amer. J. Math. 122 (2000), no. 6, 1121-1138.

[13] S. Popa, The commutant modulo the set of compact operators of a von Neumann algebras, J. Funct. Anal. 712 (1987), 393-408. 
[14] K-S. Saito, Toeplitz operators associated with analytic crossed products, Math. Proc. Cambridge Philos. Soc. 108 (1990), no. 3, 539-549.

[15] M. Takesaki, Theory of Operator Algebras. II. Encyclopaedia of Mathematical Sciences, 125. Operator Algebras and Non-commutative Geometry, 6. Springer-Verlag, Berlin, 2003.

[16] J. Xia, A characterization of compact perturbations of Toeplitz operators. Trans. Amer. Math. Soc. 361 (2009), no. 10, 5163-5175.

Graduate School of Mathematics, Kyushu University, Fukuoka 819-0395, Japan

E-mail address: ma213034@math.kyushu-u.ac.jp 